\title{
Communication
}

\section{Extraordinary Transmission and Radiation From Finite by Infinite Arrays of Slots}

\author{
Miguel Camacho $^{(\mathbb{D}}$, Rafael R. Boix ${ }^{(\mathbb{0}}$, Francisco Medina ${ }^{(\mathbb{D}}$, Alastair P. Hibbins, and J. Roy Sambles ${ }^{(}$
}

\begin{abstract}
In this communication an efficient method of moments (MoM) code is used for the analysis of the extraordinary transmission (EOT) through a periodic array of rectangular slots in a conducting screen, in the case where the number of slots is finite in one direction and infinite in the orthogonal direction. The slots can be arbitrarily rotated within the periodic unit cell. Once the magnetic current density on the slots is obtained by means of MoM, both the transmission coefficient and the far-field radiated by the array of slots are computed. The onset of EOT turns out to be strongly dependent on the orientation of the slots with respect to the direction in which the array is infinite. If the slots are perpendicular to this direction, EOT appears for a single infinite chain of slots. However, tens of parallel chains of slots are required to reproduce the EOT response when the slots are aligned along the parallel chains direction. The obtained radiation patterns show the excitation of grating lobes as the number of slots grow in the direction where the arrays are finite.
\end{abstract}

Index Terms-Arrays, moment methods, periodic structures, scattering.

\section{INTRODUCTION}

Two decades ago, experiments carried out at optical frequencies found remarkable transmission of light through periodic arrays of subwavelength holes in conducting screens at frequencies meaningfully lower than the cutoff frequency of the holes, which coined the term extraordinary (optical) transmission (EOT) [1]. The EOT phenomenon was initially explained in terms of the excitation of surface plasmons supported by the air-metal interface [2], which are significantly affected by the plasma-like behavior of electrons at optical frequencies. However, the discovery of EOT at millimeter wave frequencies [3], [4], where metals roughly behave as perfect electric conductors (PEC), made it clear EOT is not related to the properties of metals at optical frequencies, and is ultimately due to the periodicity of the arrays of holes through which the electromagnetic waves transmit [4]. Although most of the theoretical and experimental

Manuscript received January 21, 2019; revised July 8, 2019; accepted August 2, 2019. Date of publication August 22, 2019; date of current version January 3, 2020. This work was supported in part by the Engineering and Physical Sciences Research Council (EPSRC), U.K., through the EPSRC Centre for Doctoral Training in Metamaterials under Grant EP/L015331/1, in part by the Junta de Andalucía under Project P12-TIC-1435, and in part by the Spanish Ministry of Economy, Industry and Competitiveness with EU Fondo Europeo de Desarrollo Regional (FEDER) under Project TEC201784724-P. (Corresponding author: Rafael R. Boix.)

M. Camacho is with the Electromagnetic and Acoustic Materials Group, Department of Physics and Astronomy, University of Exeter, Exeter EX4 4QL, U.K., and also with the School of Engineering and Applied Sciences, University of Pennsylvania, Philadelphia, PA 19104-6391 USA (e-mail: mc586@exeter.ac.uk).

R. R. Boix and F. Medina are with the Microwaves Group, Department of Electronics and Electromagnetism, College of Physics, University of Seville, 41012 Seville, Spain (e-mail: boix@us.es; medina@us.es).

A. P. Hibbins and J. R. Sambles are with the Electromagnetic and Acoustic Materials Group, Department of Physics and Astronomy, University of Exeter, Exeter EX4 4QL, U.K. (e-mail: a.p.hibbins@exeter.ac.uk; j.r.sambles@exeter.ac.uk).

Color versions of one or more of the figures in this communication are available online at http://ieeexplore.ieee.org.

Digital Object Identifier 10.1109/TAP.2019.2935819 studies on EOT have focused on 2-D periodic arrays of holes [1]-[4], the EOT phenomenon also occurs in 1-D periodic arrays of holes as shown in [5]. In fact, linear chains of subwavelength holes can be considered as the basical geometrical unit showing this property [5]. In the last few years, the EOT phenomenon has found an application in the design of antennas [6]. An excellent review on the EOT topic can be found in [7].

Just a few years after the experimental discovery of EOT, theoretical computations on the transmission through periodic infinite arrays of holes and periodic finite arrays of holes were carried out by means of the coupled mode method [7]. In this numerical method, the fields inside the holes are expanded in terms of waveguide modes, and the fields in the air regions surrounding the conducting screen are expressed in terms of a discrete (infinite case) or continuous (truncated case) spectrum of plane waves. The coupled mode method converges very quickly with respect to the number of waveguide modes when the conducting screens are electrically thick, but it may require hundreds of waveguide modes for accurate results in the case of electrically thin conducting screens [8], since the magnetic current singularities at the holes conducting edges are not modeled in an accurate way by waveguide modes.

In this communication we explore the phenomenon of EOT through periodic arrays of tilted rectangular slots in zero thickness PEC screens in the case where the number of slots is finite in one direction, and infinite in the orthogonal direction. Other authors have studied the analysis of the scattering by finite and infinite periodic arrays of PEC rectangular patches or dipoles in free space (see [9] and references therein), and also, by finite and infinite periodic arrays of slots in a conducting screen [10]. However, the aforementioned papers focus on the scattering and radiation properties of the arrays and on their frequency selective properties, whereas, in this communication we focus on the occurrence of the EOT phenomenon. In this communication the spatial domain version of method of moments (MoM) has been used for the determination of the magnetic currents on the slots. Owing to the use of basis functions which account for the singularities of the magnetic currents at the edges of the slots [11], the MoM turns out to converge very quickly with respect to the number of basis functions per slot, which makes it possible to study large truncated arrays of slots in conducting screens of negligible thickness, as opposed to the coupled mode method. Both the use of basis functions accounting for edge singularities and the use of Ewald's method for the determination of the 1-D periodic Green's function [12], have made it possible to implement a version of MoM that outperforms the ones presented in previous papers [9], [10]. The code implemented has been used to study the phenomenon of EOT in single infinite chains of narrow rectangular apertures in different orientations (which complements the studies carried out in [5]), and to check how the EOT results obtained for a finite number of parallel chains (1-D periodic problem) converge to those obtained for an infinite number of chains (2-D periodic problem) as the number of parallel chains becomes larger. The same MoM code can also be used to obtain the dispersion relation of the leaky waves that can propagate 


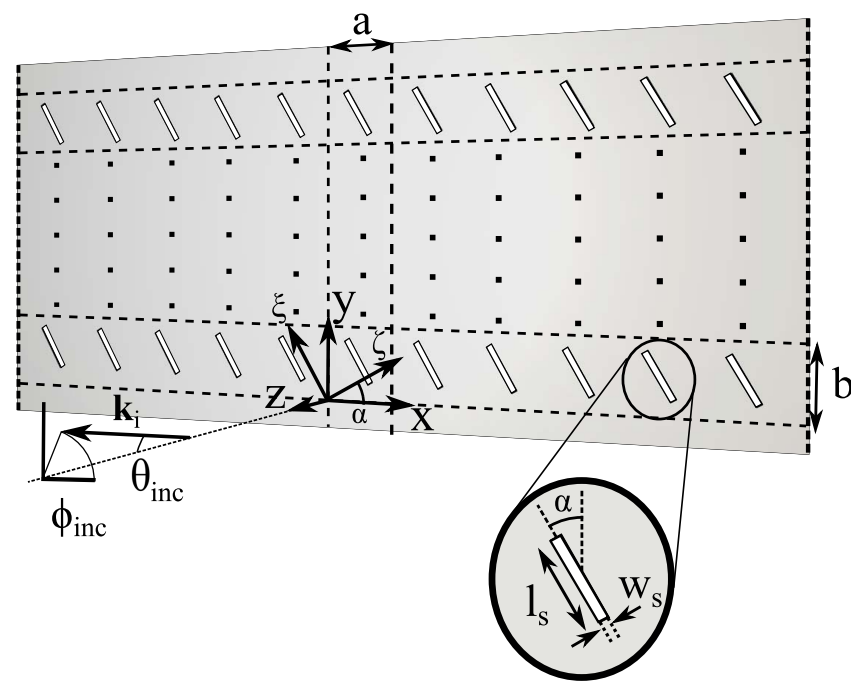

Fig. 1. Perspective view of a 1-D periodic array of rectangular holes. A finite number $N_{S}$ of slots is assumed to be located in the $y$-direction with a spacing $b$, while the number of holes in the $x$-direction is infinite with a spacing $a$. The slots are rotated an angle $\alpha$, which also defines a new set of coordinates $(\zeta, \xi)$, aligned with the axes of the slots.

through the 1-D periodic perforated screens in case Ewald's method for 1-D periodic Green's function is adequately adjusted to deal with leaky waves [13]. Also in this communication, an asymptotic expansion of the electric field has been carried out to obtain the radiation pattern of the array at large distances from the perforated screen. The results obtained for these radiation patterns show how transmitted power is split between the direction of the impinging waves and the direction of the excited grating lobes.

\section{NUMERICAL APPROACH}

Let us consider the geometry depicted in Fig. 1, in which an infinite perfectly conducting plane of negligible thickness is perforated with a periodic array of slots, which is infinite in the $x$-direction and finite in the $y$-direction. The periodic unit cell is a rectangle of dimensions $a \times b$, and $N_{S}$ unit cells are considered in the $y$-direction. The slots are centered in each unit cell, their width and length are $w_{\mathrm{s}}$ and $l_{\mathrm{S}}$, respectively, $\left(w_{s} \ll l_{s}<\min (a, b)\right)$, and they are tilted an angle $\alpha$ with respect to the $y$-axis as shown in Fig. 1 . Let the surface occupied by the periodic unit cells be $S_{i j}=\{i a \leq x \leq(i+1) a$; $j b \leq y \leq(j+1) b\}\left(i=\cdots,-1,0,1, \ldots ; j=0, \ldots, N_{s}-1\right)$, and let $\eta_{i j}$ be the surface of the slot located on $S_{i j}$.

In the following, a harmonic time dependence of the physical quantities of the type $e^{\mathrm{j} \omega t}$ will be assumed and suppressed throughout. Let us assume that a plane wave propagating in the half-space $z<0$ of Fig. 1 in a direction given by the spherical coordinates $\left(\phi_{\text {inc }}, \theta_{\text {inc }}\right)$ obliquely impinges on the perforated screen. Let $\mathbf{E}_{t}^{\mathrm{sc}}(x, y, z=0)=$ $E_{x}^{\mathrm{sc}}(x, y, z=0) \hat{\mathbf{x}}+E_{y}^{\mathrm{sc}}(x, y, z=0) \hat{\mathbf{y}}$ be the tangential scattered electric field induced on the screen $\left(\mathbf{E}_{t}^{\mathrm{sc}}(x, y, z=0)=0\right.$ on the conducting portion of the perforated screen) by the incident plane wave. Then, the functions $\mathbf{E}_{t}^{\mathrm{sc}}(x, y, z=0)$ on the $N_{s}$ slots $\eta_{0 j}$ will be the solution of the following set of $N_{S}$ coupled integral equations:

$$
\begin{gathered}
\mathbf{J}^{\mathrm{as}}(x, y)+\sum_{i=-\infty}^{+\infty} \sum_{j=0}^{N_{s}-1} \iint_{\eta_{i j}} \overline{\mathbf{G}}_{M}\left(x-x^{\prime}, y-y^{\prime}\right) \\
\cdot \mathbf{E}_{t}^{\mathrm{sc}}\left(x^{\prime}, y^{\prime}, z=0\right) d x^{\prime} d y^{\prime}=\mathbf{0} \quad(x, y) \in \eta_{0 j} \\
\left(j=0, \ldots, N_{s}-1\right)
\end{gathered}
$$

where $\mathbf{J}^{\mathrm{as}}(x, y)$ is the electric current density excited by the plane wave impinging on the conducting screen in the absence of the slots, and $\overline{\mathbf{G}}_{M}(x, y)$ is the dyadic Green's function defined in [14, Eqs. (2) and (3)]. Since $\mathbf{E}_{t}^{\mathrm{sc}}(x, y, z=0)$ is a Floquet-periodic function of $x$ of period $a$ (and therefore, once we find a solution $\mathbf{E}_{t}^{\mathrm{sc}}(x, y, z=0)$ in the slots $\eta_{0 j}\left(j=0, \ldots, N_{s}-1\right)$, the electric field on the rest of the slots of Fig. 1 will be automatically determined), (1) can be rewritten as

$$
\begin{aligned}
\mathbf{J}^{\mathrm{as}}(x, y)+\sum_{j=0}^{N_{s}-1} \iint_{\eta_{0 j}} \overline{\mathbf{G}}_{1 \mathrm{D}}^{\mathrm{per}}\left(x-x^{\prime}, y-y^{\prime}\right) \\
\cdot \mathbf{E}_{t}^{\mathrm{sc}}\left(x^{\prime}, y^{\prime}, z=0\right) d x^{\prime} d y^{\prime}=\mathbf{0} \quad(x, y) \in \eta_{0 j} \\
\left(j=0, \ldots, N_{s}-1\right)
\end{aligned}
$$

where $\overline{\mathbf{G}}_{1 \mathrm{D}}^{\mathrm{per}}(x, y)$ is the 1-D periodic dyadic Green's function given by

$$
\overline{\mathbf{G}}_{1 \mathrm{D}}^{\mathrm{per}}(x, y)=\sum_{i=-\infty}^{+\infty} \overline{\mathbf{G}}_{M}(x-i a, y) \mathrm{e}^{\mathrm{j} i k_{x 0} a}
$$

and $k_{x 0}=-k_{0} \sin \left(\theta_{\text {inc }}\right) \cos \left(\phi_{\text {inc }}\right)\left(k_{0}=\omega \sqrt{\mu_{0} \epsilon_{0}}=2 \pi / \lambda_{0}, \lambda_{0}\right.$ being the free space wavelength). In order to determine the value of $\mathbf{E}_{t}^{\mathrm{sc}}(x, y, z=0)$ in the slots $\eta_{0 j}\left(j=0, \ldots, N_{s}-1\right)$, we have applied Galerkin's version of MoM to the set of $N_{s}$ integral equations of (2). The basis functions for $\mathbf{E}_{t}^{\mathrm{sc}}(x, y, z=0)$ have been chosen in such a way that the corresponding basis functions for the magnetic current density on the slots, $\mathbf{M}^{\mathrm{sc}}(x, y)=\hat{\mathbf{z}} \times \mathbf{E}_{t}^{\mathrm{sc}}(x, y, z=0)$, coincide with the basis functions used in [15] to approximate the electric current density on the rectangular dipoles of a multilayered periodic structure (see [15, Eqs. (17)-(19)]). These basis functions have the advantage that they account for the physical edge singularities of the magnetic current density at the slot edges, and therefore, they ensure a fast convergence of MoM with respect to the number of basis functions as shown in [15, Fig. 8]. For the particular problem treated in this communication, numerical simulations have shown that only four basis functions per slot suffice to provide very accurate results in the MoM solution of (2). This is checked in Section III where our results are compared with CST results, and a good agreement is found.

The MoM matrix entries have been computed in a very efficient way. In particular, these entries have been expressed as double integrals in a rectangular domain with respect to the two coordinates $\zeta$ and $\xi$ shown in Fig. 1. The integrand of these integrals are products of cross-correlations between the basis functions (or between their divergences) times the free-space scalar 1-D periodic Green's function (see [14, Eqs. (43)-(45)]). The scalar 1-D periodic Green's function (different from the Green's functions of [14]) has been efficiently obtained by means of Ewald's method as explained in [12, Section IV]. For large separations between source and field points $\left(R_{\mathrm{t}}\right.$ in [12]), where the spectral series of Ewald's method may show numerical problems, we have used the spectral representation of the 1-D periodic Green's function for its computation (see [12, Eq. (34)]). The singularities of the integrands of the double integrals have been handled as explained in [14].

If we solve the wave equation in the half-space $z>0$ of Fig. 1 , it can be shown that the electric field scattered by the perforated conducting screen in that half-space can be written as

$$
\begin{array}{r}
\mathbf{E}^{\mathrm{Sc}}(x, y, z>0)=\frac{1}{2 \pi} \sum_{m=-\infty}^{+\infty} \int_{-\infty}^{+\infty} \widetilde{\mathbf{E}}^{\mathrm{sc}}\left(k_{x m}, k_{y}, z=+0\right) \\
\times \mathrm{e}^{\mathrm{j}\left(k_{x m} x+k_{y} y-k_{z m} z\right)} d k_{y}
\end{array}
$$

where $k_{x m}=(2 \pi m / a)+k_{x 0}, k_{z m}=\sqrt{k_{0}^{2}-k_{x m}^{2}-k_{y}^{2}}$ when $k_{x m}^{2}+k_{y}^{2}<k_{0}^{2}$ and $k_{z m}=-\mathrm{j} \sqrt{k_{x m}^{2}+k_{y}^{2}-k_{0}^{2}}$ when $k_{x m}^{2}+k_{y}^{2}>k_{0}^{2}$, and where $\widetilde{\mathbf{E}}^{\mathrm{sc}}\left(k_{x m}, k_{y}, z=+0\right)$ is the 2-D Fourier transform, 
discrete in $x$ and continuous in $y$, of $\mathbf{E}^{\mathrm{sc}}(x, y, z=+0)$. If we introduce spherical coordinates in (4), the electric field radiated by the dominant $m=0$ Floquet mode of (4) (the only radiative mode when $a<\lambda_{0}$ ) will be given by

$$
\begin{aligned}
\mathbf{E}^{\mathrm{sc}, 0}(r \gg, \theta, \phi) & \left.\right|_{Z>0}=\frac{1}{2 \pi} \mathrm{e}^{\mathrm{j} k_{x} r \sin \theta \cos \phi} \int_{-\infty}^{+\infty} \widetilde{\mathbf{E}}^{\mathrm{sc}}\left(k_{x} 0, k_{y}, z=+0\right) \\
& \times\left.\mathrm{e}^{\mathrm{j}\left(k_{y} \sin \theta \sin \phi-\sqrt{k_{0}^{2}-k_{x 0}^{2}-k_{y}^{2}} \cos \theta\right) r} d k_{y}\right|_{r \gg}
\end{aligned}
$$

Now, if we carry out an asymptotic evaluation of the integral of (5) by means of Rayleigh's method of stationary phase (see [16, pp. 284-286]), it turns out that

$$
\begin{aligned}
\left.\mathbf{E}^{\mathrm{sc}, 0}(r \gg, \theta, \phi)\right|_{\mathrm{z}>0} \approx & \frac{\mathrm{e}^{\mathrm{j}\left(k_{x 0} \sin \theta \cos \phi-\beta_{p s}(\theta, \phi)\right) r} \cos \theta}{\sqrt{2 \pi r\left(1-\sin ^{2} \theta \cos ^{2} \phi\right)}} \\
& \times \sqrt{\mathrm{j} k_{0}}\left(\frac{1-\sin ^{2} \theta_{\text {inc }} \cos ^{2} \phi_{\text {inc }}}{1-\sin ^{2} \theta \cos ^{2} \phi}\right)^{1 / 4} \\
& \times \widetilde{\mathbf{E}}^{\mathrm{sc}}\left(k_{x}, k_{y}=-k_{y}^{p s}(\theta, \phi), z=+0\right)
\end{aligned}
$$

where

$$
\beta_{p s}(\theta, \phi)=k_{0} \sqrt{\left(1-\sin ^{2} \theta_{\text {inc }} \cos ^{2} \phi_{\text {inc }}\right)\left(1-\sin ^{2} \theta \cos ^{2} \phi\right)}
$$

and

$$
k_{y}^{p s}(\theta, \phi)=k_{0} \sin \theta \sin \phi \sqrt{\frac{\left(1-\sin ^{2} \theta_{\mathrm{inc}} \cos ^{2} \phi_{\mathrm{inc}}\right)}{1-\sin ^{2} \theta \cos ^{2} \phi}} .
$$

When (4) is used in the equation $\nabla \cdot \mathbf{E}^{\mathrm{sc}}(x, y, z>0)=0$, the $z$ component of the vector quantity $\widetilde{\mathbf{E}}^{\mathrm{sc}}\left(k_{x 0}, k_{y}, z=+0\right)$ appearing in (6) can be expressed in terms of the two components of $\widetilde{\mathbf{E}}_{t}^{\mathrm{sc}}\left(k_{x 0}, k_{y}, z=0\right)$ as shown below

$$
\begin{aligned}
& \widetilde{E}_{z}^{\mathrm{sc}}\left(k_{x 0}, k_{y}, z=+0\right) \\
& \quad=\frac{1}{k_{z 0}}\left(k_{x 0} \widetilde{E}_{x}^{\mathrm{sc}}\left(k_{x 0}, k_{y}, z=0\right)+k_{y} \widetilde{E}_{y}^{\mathrm{sc}}\left(k_{x 0}, k_{y}, z=0\right)\right) .
\end{aligned}
$$

Once MoM is applied, $\widetilde{\mathbf{E}}_{t}^{\mathrm{sc}}\left(k_{x 0}, k_{y}, z=0\right)$ can be obtained as the 2-D Fourier transform, discrete in $x$ and continuous in $y$, of $\mathbf{E}_{t}^{\mathrm{sc}}(x, y, z=0)$. This fact, together with (9), implies that the solution of (2) makes it possible to compute the far-field $\left.\mathbf{E}^{\mathrm{sc}, 0}(r \gg, \theta, \phi)\right|_{z>0}$ by means of (6).

For the wave impinging on the perforated screen of Fig. 1, we can define the scattering width [17] of the dominant $m=0$ Floquet mode along the incidence direction as

$$
\begin{aligned}
\sigma_{1 \mathrm{D}}^{0}\left(\theta_{\text {inc }}, \phi_{\text {inc }}\right) & =\lim _{r \rightarrow \infty} \frac{2 \pi \sqrt{y^{2}+z^{2}}\left|\mathbf{E}^{\mathrm{sc}, 0}\left(r, \theta=\theta_{\text {inc }}, \phi=\phi_{\text {inc }}\right)\right|^{2}}{\left|\mathbf{E}_{0}\right|^{2}} \\
& =\frac{k_{0} \cos ^{2} \theta_{\text {inc }}\left|\widetilde{\mathbf{E}}^{\mathrm{sc}}\left(k_{x 0}, k_{y}=k_{y 0}, z=+0\right)\right|^{2}}{\left(1-\sin ^{2} \theta_{\text {inc }} \cos ^{2} \phi_{\text {inc }}\right)^{1 / 2}\left|\mathbf{E}_{0}\right|^{2}}
\end{aligned}
$$

where we have made use of (6). The quantity $\sigma_{1 \mathrm{D}}^{0}$ of (10) will be a measure of the length in the $y$-direction that the truncated array of slots of Fig. 1 presents to a wave impinging on the array.

In order to estimate the amount of power transmitted through the array of holes of Fig. 1, we are going to define a dimensionless transmission coefficient, $T_{1 \mathrm{D}}$, as the ratio between the power radiated into the half-space $z>0$ by the $N_{S}$ unit cells located within the interval $0<x<a,\left.P_{\mathrm{rad}}^{1 \mathrm{D}}\right|_{z>0}$, and the power of the impinging plane wave available at those $N_{S}$ cells, $P_{\mathrm{av}}^{1 \mathrm{D}}$, that is,

$$
T_{1 \mathrm{D}}=\frac{\left.P_{\mathrm{rad}}^{1 \mathrm{D}}\right|_{\mathrm{Z}>0}}{P_{\mathrm{av}}^{1 \mathrm{D}}} .
$$

The quantitiy $\left.P_{\mathrm{av}}^{1 \mathrm{D}}\right|_{z>0}$ of (11) is given by

$$
P_{\mathrm{av}}^{1 \mathrm{D}}=\frac{N_{S} a b \cos \theta_{\mathrm{inc}}\left|\mathbf{E}_{0}\right|^{2}}{2 Z_{0}}
$$

where $Z_{0}=\sqrt{\mu_{0} / \epsilon_{0}}$ is the free space wave impedance, and where $\mathbf{E}_{0}$ is the complex vector amplitude of the electric field of the impinging plane wave. The quantity $P_{\mathrm{rad}}^{1 \mathrm{D}}$ of (11) is given by

$$
\begin{gathered}
\left.P_{\mathrm{rad}}^{1 \mathrm{D}}\right|_{z>0}=\frac{1}{2} \operatorname{Re}\left\{\sum _ { j = 0 } ^ { N _ { s } - 1 } \int \int _ { \eta _ { 0 j } } \left[\mathbf{E}^{\mathrm{sc}}(x, y, z=+0)\right.\right. \\
\left.\left.\times\left(\mathbf{H}^{\mathrm{sc}}(x, y, z=+0)\right)^{*}\right] \cdot \hat{\mathbf{z}} d x d y\right\}
\end{gathered}
$$

where $\mathbf{H}^{\mathrm{sc}}(x, y, z)$ stands for the magnetic field scattered by the perforated conducting screen. After some mathematical manipulations, it is possible to express $\left.P_{\mathrm{rad}}^{1 \mathrm{D}}\right|_{z>0}$ in a simple way in terms of the weight coefficients of the basis functions of $\mathbf{E}_{t}^{\mathrm{sc}}(x, y, z=0)$, and in terms of the MoM matrix entries.

\section{NUMERICAL RESULTS}

Fig. 2 shows the frequency dependence of the normalized scattering width of single infinite chains of slots for different orientations of the slots with respect to the periodicity direction. The direction of the electric field of the impinging wave has always been taken perpendicular to the slots direction. Our results obtained with the MoM approach described in Section II for the normalized scattering width are compared in Fig. 2 with results obtained by means of the commercial software CST [18] for the case $l_{S}=0.4 a$. Excellent agreement is found between both sets of results. We have found that the CPU time required by CST is around 200 times larger than that required by our in-house software, and this CPU time ratio excludes the CPU time required for adaptive-mesh convergence with CST. The important CPU time saving clearly shows the advantages of developing an efficient in-house software for the study of EOT phenomena in truncated periodic structures.

Please note that a Wood's anomaly, consisting of a zero in transmission at $a / \lambda_{0}=1$, is found in Fig. 2(b) and (c). The Wood's anomaly is associated with the onset of "grating lobes" that propagate along the plane of the array at this frequency, with a wavelength equal to the periodicity. It is preceded by an associated EOT peak (maximum of transmission) for $l_{s} / a=0.4$ as expected [4], [14]. However, the Wood's anomaly and the EOT peak are not present in Fig. 2(a). In order to explain the lack of Wood's anomaly in Fig. 2(a) from a physical point of view, we have to think that the Wood's anomaly at $a / \lambda_{0}=1$ is connected with the onset of the grating lobes $m= \pm 1$ scattered by the periodic structure of Fig. 1 [14]. In the case of Fig. 2(a), the planes $x=q a(q=\cdots,-1,0,+1, \ldots)$ act as magnetic walls, and for the particular direction of the excitation electric field in Fig. 2(a), the two grating lobes $m= \pm 1$ launched when $a / \lambda_{0} \geq 1$ merge to form a $\mathrm{TE}_{2}$ mode that propagates along an equivalent parallel-plate waveguide limited by two magnetic walls at $x=0$ and $x=a$. However, TE modes in the equivalent parallel-plate waveguide do not lead to Wood's anomalies and EOT peaks, which is explained in detail in [4] by means of an equivalent circuit containing frequency-dependent capacitances and inductances. Note that the Wood's anomaly occurs in Fig. 2(c) for $a / \lambda_{0}=1$ (when the axis of the slot is orthogonal to the direction of the periodicity) in spite of the apparent similarity with the case of Fig. 2(a). This is because in the former case the planes $x=q a(q=\cdots,-1,0,+1, \ldots)$ act as electric walls, and for the particular direction of the excitation electric field in Fig. 2(c), the two grating lobes $m= \pm 1$ launched when 
(a)
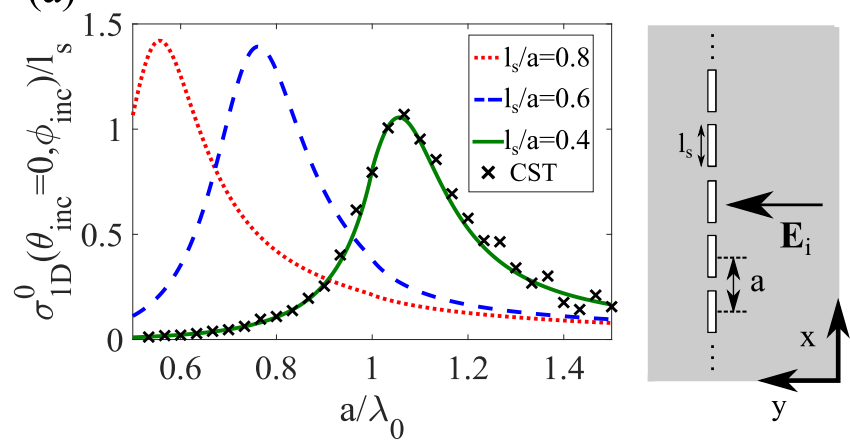

(b)
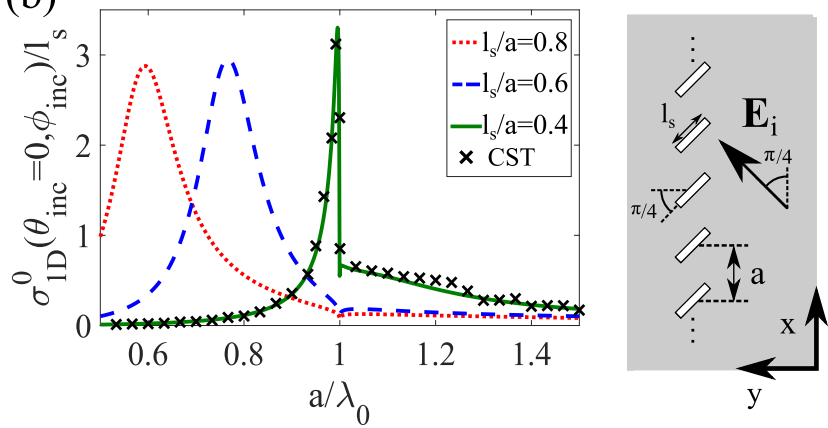

(c)
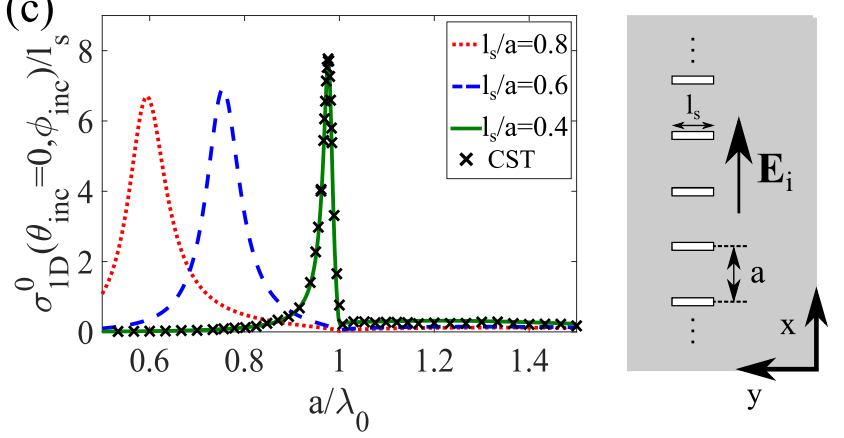

Fig. 2. Normalized scattering width of single infinite periodic chains of slots $\left(N_{S}=1\right.$ in Fig. 1) for different values of the slots length under normal incidence conditions. Results are presented for (a) slots parallel to the periodicity direction, $\alpha=90^{\circ}$, (b) slots tilted an angle $\alpha=45^{\circ}$ with respect to the periodicity direction, and (c) slots perpendicular to the periodicity direction, $\alpha=0^{\circ}$. Our results obtained with MoM for the case $l_{s} / a=0.4$ (solid lines) are compared with CST results $(\times)$. Parameters: $w_{s} / a=0.05$, $\theta_{\text {inc }}=0^{\circ}$.

$a / \lambda_{0} \geq 1$ merge to form a $\mathrm{TM}_{2}$ mode that should propagate along an equivalent parallel-plate waveguide limited by two electric walls at $x=0$ and $x=a$. And TM modes in the equivalent waveguide do generate Wood's anomalies and EOT peaks as explained in detail in [4], which justifies the presence of the Wood's anomaly when $a / \lambda_{0}=1$ in Fig. 2(c). Finally, in the case of Fig. 2(b), there are no symmetry conditions at the onset of grating lobes (no electric or magnetic walls at the planes $x=q a$ ) that prevent the excitation of TM modes in the equivalent waveguide, and the Wood's anomaly at $a / \lambda_{0}=1$ is present.

We have carried out CST simulations of the periodic structures of Fig. 2 for $l_{S} / a=0.4$ in the case where the width of the conducting plane of Fig. 1 is finite in the $y$-direction. The CST results obtained for the normalized scattering width of the array in that case practically coincide with those of Fig. 2, provided, the edges of the conducting plane in the $y$-direction are further than three periods from the array.


Fig. 3. Transmission coefficient of $N_{s}$ parallel infinite periodic chains of slots for different values of $N_{S}$ under normal incidence conditions. Results are presented for (a) slots parallel to the periodicity direction, $\alpha=90^{\circ}$, (b) slots tilted an angle $\alpha=45^{\circ}$ with respect to the periodicity direction, and (c) slots perpendicular to the periodicity direction, $\alpha=0^{\circ}$. Parameters: $l_{s} / a=0.4$, $w_{s} / a=0.05, b=1.1 a, \theta_{\text {inc }}=0^{\circ}$.

This can serve as a guide for realistic applications of the phenomena presented here.

The implemented MoM code has also been used to obtain the dispersion relation of waves traveling along the $x$-direction in the periodic structure of Fig. 2(b) when $l_{s} / a=0.4$. For that purpose, Ewald's method for the determination of the scalar 1-D periodic Green's function has been modified to account for complex wavenumbers along the $x$-direction as described in [13]. The results obtained indicate that in the neighborhood of the EOT peak, a backward proper leaky wave can propagate along the periodic chain of slots, and that the leaky wave radiates at broadside direction when $a / \lambda_{0}=0.995$, which exactly corresponds to the frequency of the EOT peak appearing in Fig. 2(b). This finding connects with the surface plasmon explanation of the EOT phenomenon described in [2]. Unfortunately, the dispersion relation results cannot be shown in this communication due to size limitations.

In Fig. 3 we plot results for the transmission coefficient $T_{1 \mathrm{D}}$ of $N_{S}$ parallel infinite chains of slots in a periodic configuration as shown in Fig. 1. The period in the $y$-direction where the periodic array is finite, $b=1.1 a$, is chosen to be different from the period in 
(a)

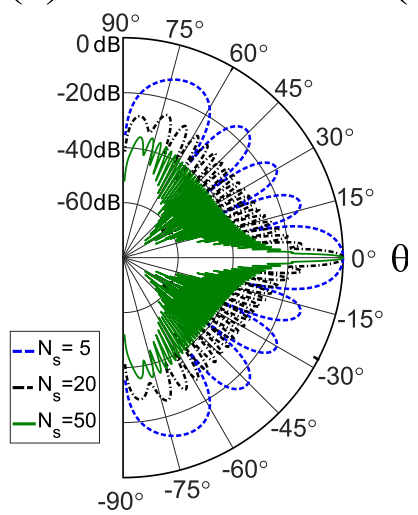

(b)

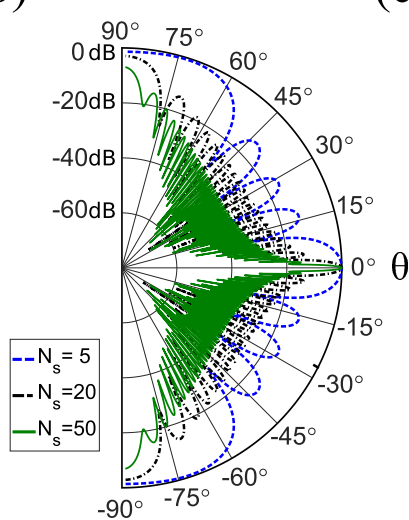

(c)

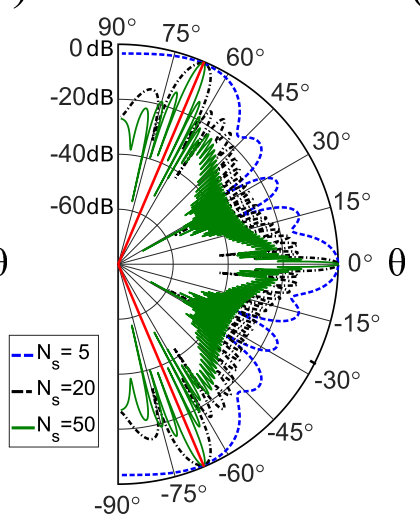

(d)

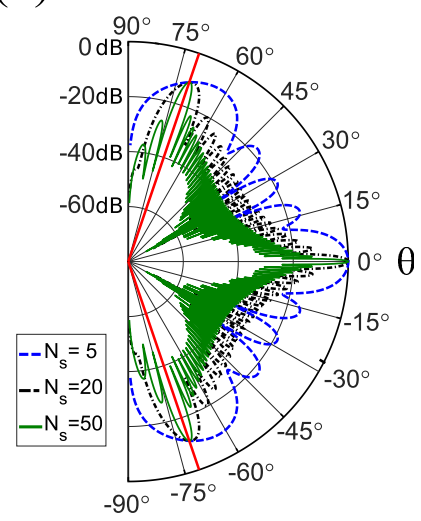

Fig. 4. Normalized radiation patterns in the $y z$ plane for $z>0\left(\phi= \pm 90^{\circ}\right)$ of the periodic arrays of slots analyzed in Fig. 3 when (a) $\alpha=90^{\circ}$ and $a / \lambda_{0}=0.887$, (b) $\alpha=45^{\circ}$ and $a / \lambda_{0}=0.897$, (c) $\alpha=45^{\circ}$ and $a / \lambda_{0}=0.995$, and (d) $\alpha=00^{\circ}$ and $a / \lambda_{0}=0.960$. The direction of the grating lobes is indicated in red. Parameters: $l_{s} / a=0.4, w_{s} / a=0.05, b=1.1 a, \theta_{\text {inc }}=0^{\circ}$.

the $x$-direction where the array is infinite, $a$, so that the different phenomena related to the periodicity in each direction can be clearly distinguished. By comparison with Fig. 2, in Fig. 3 we have restricted ourselves to the case where $l_{s} / a=0.4$, since this is the case for which EOT peaks are expected to appear according to Fig. 2. Note that in Fig. 3(a) a Wood's anomaly starts being apparent when $N_{s} \geq 20$ for $b / \lambda_{0}=1$ (which corresponds to $a / \lambda_{0}=0.91$ in the figure), and the associated EOT peak also becomes observable. This Wood's anomaly would correspond to the onset of the grating lobes $m=0, n= \pm 1$ if the periodic array of slots were infinite in the $x$ - and $y$-directions (as in the case treated in Section II of [14]). In accordance with the explanation given in the previous paragraph for the results of Fig. 2(c), this Wood's anomaly is allowed by the structure of Fig. 3(a) because the slots are perpendicular to the $y$ direction, and as the number of slots in the $y$-direction grows and the periodicity in this direction becomes more defined, the phenomena associated with this periodicity (Wood's anomaly and EOT peak) become observable in Fig. 3(a). The Wood's anomaly for $a / \lambda_{0}=1$ is not present in Fig. 3(a) for the same symmetry arguments introduced in the comments to Fig. 2(a). In Fig. 3(c) the Wood's anomaly for $a / \lambda_{0}=1$ and the associated EOT peak appear for all values of $N_{S}$ since the symmetry arguments used to explain the Wood's anomaly of Fig. 2(c) still hold for Fig. 3(c). However, the Wood's anomaly for $b / \lambda_{0}=1$ is absent because the slots in the structure of Fig. 3(c) are parallel to the $y$-direction, and as this structure becomes periodic in this direction, the grating lobes associated with this periodicity direction will not be excited when $b / \lambda_{0}=1$ for the same symmetry reasons the grating lobes associated with the periodicity in the $x$-direction were not excited in Fig. 2(a) when $a / \lambda_{0}=1$. Concerning Fig. 3(b), since the structure analyzed in this latter figure is not symmetric, the two Wood's anomalies for $b / \lambda_{0}=1$ and for $a / \lambda_{0}=1$ and the associated EOT peaks are present in the results plotted. However, whereas the Wood's anomaly for $a / \lambda_{0}=1$ is present for all values of $N_{s}$, the Wood's anomaly for $b / \lambda_{0}=1$ becomes noticeable for $N_{S} \geq 20$. This is due to the fact that the Wood's anomaly for $a / \lambda_{0}=1$ is related to the periodicity in the $x$-direction, which exists for all values of $N_{s}$, the Wood's anomaly for $b / \lambda_{0}=1$ is related to the periodicity in the $y$-direction, which requires a large value of $N_{s}$ to start being apparent. Please note that when $N_{S} \geq 20$, an additional Wood's anomaly is formed in Fig. 3(a)-(c) when $a / \lambda_{0}=1.35$, which corresponds to the case where the relation $(1 / a)^{2}+(1 / b)^{2}=\left(1 / \lambda_{0}\right)^{2}$ fulfills. This latter Wood's anomaly would exactly correspond to the onset of the grating lobes $m= \pm 1, n= \pm 1$ if the periodic array of slots were infinite both in the $x$-and $y$-directions, and therefore, it is something to be expected as the array of slots increases its size in the $y$-direction.

In Fig. 4 we have represented the normalized power radiation patterns emitted by the truncated array of slots of Fig. 1 in the $y z$ plane at the EOT peaks of Fig. 3(a)-(c). Since there are two EOT peaks in Fig. 3(b) (one for $a / \lambda_{0}=0.897$ and one for $a / \lambda_{0}=0.995$ ), two different radiation patterns are plotted in this case, one for each EOT peak. Note that as $N_{S}$ increases, the radiation of the dominant $m=0$ Floquet mode along the incidence direction $\left(\theta=0^{\circ}\right)$ becomes more and more directive. Of course, this would be the only radiation direction in case the array were infinite in the $y$-direction. In the cases of Fig. 4(c) and (d), one can see the excitation of two grating lobes that would correspond to the grating lobes $m=0, n= \pm 1$ if the periodic array of slots were infinite in the $x$ - and $y$-directions. In this latter case, the directions of radiation of these two grating lobes with respect to the positive $z$-axis would be given by the angles

$$
\theta_{g l}^{0, \pm 1}= \pm \arctan \left(\frac{\lambda_{0} / b}{\sqrt{1-\left(\lambda_{0} / b\right)^{2}}}\right) .
$$

The angles predicted by (14) are marked in red in Fig. 4(c) and (d) $\left[\theta_{g l}^{0, \pm 1}= \pm 66.06^{\circ}\right.$ in the case of Fig. 4(c) and $\theta_{g l}^{0, \pm 1}= \pm 71.26^{\circ}$ in the case of Fig. 4(d)], and it can be checked that these two angles coincide with the radiation maxima related to the grating lobes when $N_{S} \geq 20$, which provides an indirect validation for the computed radiation patterns.

\section{CONCLUSION}

An efficient MoM code has been implemented for the analysis of EOT in periodic arrays of tilted slots which are infinite in one direction and truncated in the orthogonal direction. Good agreement has been found between our results and CST, with our code being around 200 times faster than CST. The results obtained indicate even though EOT peaks and Wood's anomalies may not reveal in single chains of infinite slots owing to the lack of periodicity in the direction perpendicular to the chains, they become apparent when more than twenty of these chains are periodically gathered. An asymptotic expansion of the fields scattered by the arrays has been carried out to compute the far-field radiation patterns These radiation patterns clearly show the excitation of grating lobes as radiation maxima that become sharper and sharper as the number of slots grows in the direction where the arrays are finite, 
irrespective of the existence or not of a Wood's anomaly at their onset.

\section{REFERENCES}

[1] T. W. Ebbesen, H. J. Lezec, H. F. Ghaemi, T. Thio, and P. A. Wolff, "Extraordinary optical transmission through sub-wavelength hole arrays," Nature, vol. 391, pp. 667-669, Feb. 1998.

[2] H. F. Ghaemi, T. Thio, D. E. Grupp, T. W. Ebbesen, and H. J. Lezec, "Surface plasmons enhance optical transmission through subwavelength holes," Phys. Rev. B, Condens. Matter, vol. 58, no. 11, pp. 6779-6782, Sep. 1998.

[3] M. Beruete et al., "Enhanced millimeter wave transmission through quasioptical subwavelength perforated plates," IEEE Trans. Antennas Propag., vol. 53, no. 6, pp. 1897-1903, Jun. 2005.

[4] F. Medina, F. Mesa, and R. Marques, "Extraordinary transmission through arrays of electrically small holes from a circuit theory perspective," IEEE Trans. Microw. Theory Techn., vol. 56, no. 12, pp. 3108-3120, Dec. 2008.

[5] J. Bravo-Abad, F. J. García-Vidal, and L. Martín-Moreno, "Resonant transmission of light through finite chains of subwavelength holes in a metallic film," Phys. Rev. Lett., vol. 93, Nov. 2004, Art. no. 227401.

[6] M. Beruete et al., "Enhanced gain by double-periodic stacked subwavelength hole array," IEEE Microw. Wireless Compon. Lett., vol. 17, no. 12, pp. 831-833, Dec. 2007.

[7] F. J. García-Vidal, L. Martín-Moreno, T. W. Ebbesen, and L. Kuipers, "Light passing through subwavelength apertures," Rev. Mod. Phys., vol. 82, no. 1, pp. 729-787, Mar. 2010.

[8] A. Yu. Nikitin, D. Zueco, F. J. García-Vidal, and L. Martín-Moreno, "Electromagnetic wave transmission through a small hole in a perfect electric conductor of finite thickness," Phys. Rev. B, Condens. Matter, vol. 78 , Oct. 2008, Art. no. 165429.
[9] J. Usoff and B. A. Munk, "Edge effects of truncated periodic surfaces of thin wire elements," IEEE Trans. Antennas Propag., vol. 42, no. 7, pp. 946-953, Jul. 1994.

[10] R. W. Scharstein, "Mutual coupling in a slotted phased array, infinite in E-plane and finite in H-plane," IEEE Trans. Antennas Propag., vol. 38, no. 8, pp. 1186-1191, Aug. 1990.

[11] A. M. Lerer and A. G. Schuchinsky, "Full-wave analysis of threedimensional planar structures," IEEE Trans. Microw. Theory Techn. vol. 41, no. 11, pp. 2002-2015, Nov. 1993.

[12] G. Valerio, P. Baccarelli, P. Burghignoli, and A. Galli, "Comparative analysis of acceleration techniques for 2-D and 3-D green's functions in periodic structures along one and two directions," IEEE Trans. Antennas Propag., vol. 55, no. 6, pp. 1630-1643, Jun. 2007.

[13] V. R. Komanduri, D. R. Jackson, F. Capolino, and D. R. Wilton, "1-D periodic green's function for leaky and complex waves using the Ewald method," IEEE Trans. Antennas Propag., vol. 64, no. 11, pp. 4703-4712, Nov. 2016.

[14] M. Camacho, R. R. Boix, and F. Medina, "Computationally efficient analysis of extraordinary optical transmission through infinite and truncated subwavelength hole arrays," Phys. Rev. E, Stat. Phys. Plasmas Fluids Relat. Interdiscip. Top., vol. 93, Jun. 2016, Art. no. 063312.

[15] R. Florencio, R. R. Boix, J. A. Encinar, and G. Toso, "Optimized periodic MoM for the analysis and design of dual polarization multilayered reflectarray antennas made of dipoles," IEEE Trans. Antennas Propag., vol. 65 , no. 7, pp. 3623-3637, Jul. 2017.

[16] R. E. Collin, Antennas and Radiowave propagation. New York, NY, USA: McGraw-Hill, 1985.

[17] C. A. Balanis, Advanced Engineering Electromagnetics. Hoboken, NJ, USA: Wiley, 1989.

[18] CST Studio Suite 3D EM Analysis Software SIMULIA by Dassault Systèmes. Accessed: Aug. 27, 2019. [Online]. Available: https://www.3ds. com/products-services/simulia/products/cst-studio-suite/ 